\title{
Avaliação da Depressão em Funcionários de uma Instituição de Ensino Superior Particilar em Vitória da Conquista - BA
}

\author{
Daiana Cirqueira Oliveira ${ }^{1}$; Matheus Lemos Silva ${ }^{2}$; Fernanda de Jesus Soares ${ }^{3}$; \\ Sara Costa Sampaio ${ }^{4}$; Felipe Oliveira Bittencourt ${ }^{5}$; Stênio Fernando Pimentel Duarte ${ }^{6}$
}

\begin{abstract}
Resumo: Boa parte das pessoas, no Brasil, tem sofrido com estados de descontrole emocional, como perturbação emocional acarretando alterações comportamentais, psicológicas e fisiológicas. Sendo assim, o objetivo desse estudo foi verificar o índice de funcionários acometidos por depressão em uma instituição de ensino superior em Vitória da Conquista- BA. Coube a esta pesquisa realizar um estudo do tipo analítico com delineamento transversal e abordagem quantitativa e teve como amostra 180 indivíduos do município de Vitória da Conquista- BA. Identificou-se que os funcionários participantes são 54,4\% mulheres e 45,6\% homens com idade média de 37,83 anos. Com relação a classe social, $70 \%$ dos funcionários se autodeclararam como pertencentes à classe $\mathrm{E}$. Apreendeu-se que quase metade deles apresentam quadro depressivo. Quanto a frequência de hipertensão, os dados mostram que $87,8 \%$ não são hipertensos; ao tabagismo, 18,3\% não fumantes e 76,1\% estiveram ausentes no sistema, não responderam a esta questão. Quanto a prática de atividades físicas, $56,1 \%$ são sedentários. As questões respondidas possibilitaram chegar à conclusão de que a qualidade de vida da maioria é normal, enquanto somente $12,8 \%$ apresentaram nível de qualidade de vida ruim. Os resultados indicam que quase metade dos funcionários possuem algum nível de depressão, assim é importante estar atento, pois qualquer nível de depressão deve impulsionar a busca de tratamento adequado. Neste trato, apreendemos que exercícios físicos estão ligados à prevenção e tratamento dos pacientes depressivos.
\end{abstract}

Palavras-Chave: Depressão. Qualidade de Vida. Saúde.

\section{Depression Evaluation in Employees of an Institution of Private Higher Education in Vitória da Conquista City - BA}

\begin{abstract}
Most people in Brazil have suffered from states of emotional discontent, such as emotional disturbance leading to behavioral, psychological and physiological changes. Therefore, the objective of this study was to verify the index of employees affected by depression in a higher education institution in Vitória da Conquista-BA. It was the responsibility of this research to carry out an analytical type study with a cross-sectional design and quantitative approach and had as sample 180 individuals from the city of Vitória da Conquista-BA. It was identified that the participating employees are $54.4 \%$ women and $45.6 \%$ men with an average age of 37.83 years. Regarding social class, $70 \%$ of the employees declared themselves as belonging to class E. It was found that almost half of them presented depressive symptoms. Regarding the frequency of hypertension, the data show that $87.8 \%$ are not hypertensive; To smoking, $18.3 \%$ were non-smokers and $76.1 \%$ were absent from the system, they did not respond to this question. As for the practice of physical activities, $56.1 \%$ are sedentary. The questions answered led to the conclusion that the quality of life of the majority is normal, while only $12.8 \%$ presented a poor quality of life. The results indicate that almost half of the employees have some degree of depression, so it is important to be aware because any level of depression should drive the search for appropriate treatment. In this treatment, we learn that physical exercises are linked to the prevention and treatment of depressive patients.
\end{abstract}

Keywords: Depression. Quality of life. Cheers.

\footnotetext{
${ }^{1}$ Graduanda do Curso de Farmácia da Faculdade Independente do Nordeste - FAINOR. E-mail: daicirqueira@ hotmail.com

${ }^{2}$ Graduando do Curso de Nutrição da Faculdade Tecnologia e Ciências - FTC. E-mail: matheus.nutri3@ gmail.com

${ }^{3}$ Graduanda do Curso de Farmácia da Faculdade Independente do Nordeste - FAINOR. E-mail: feeh_23@hotmail.com

${ }^{4}$ Graduanda do Curso de Farmácia da Faculdade Independente do Nordeste - FAINOR. E-mail: sarasampaio27@yahool.com.br

${ }^{5}$ Graduação em Farmácia, especialização em farmácia Magistral e Farmácia Hospitalar e mestrado em Saúde e Ambiente pela Universidade Tiradentes - UNIT, atualmente é docente do Colegiado de Farmácia da Faculdade Independente do Nordeste - FAINOR, Vitória da Conquista/BA. E-mail: farmaciafainor@hotmail.com

${ }^{6}$ Graduação em Ciências Biológica, Mestrado e Doutorado em Fisiopatologia Clínica e Experimental pela Universidade do Estado do Rio de Janeiro - UERJ, atualmente é docente da Faculdade Independente do Nordeste e Faculdade Tecnologia e Ciências, Vitória da Conquista Bahia. E-mail: steniofernando@gmail.com
} 


\section{Introdução}

Os transtornos mentais, em geral impactam o índice de morbidade, prejuízos na utilidade do sujeito e na qualidade de vida do sujeito acometido por este tipo de enfermidade (LIMA; FLECK, 2009). A depressão é vista como um problema de saúde pública e, considerada um dos distúrbios psiquiátricos com alta prevalência comprometendo a saúde de pelo menos 121 milhões de pessoas, conforme dados da Organização Mundial de Saúde (OMS) (APOSTOLO et al., 2011; PITCHOT et al., 2010; KAWAMURA et al, 2007).

Estudos epidemiológicos preveem que daqui a 20 anos a depressão será a segunda maior causa de doenças no mundo, perdendo tão-somente para as doenças do sistema cardiovascular (NASCIMENTO; BRITO; SANTOS, 2013). Desse modo, no Brasil, pelo menos 54 milhões de pessoas apresentarão algum nível de depressão, em algum momento de suas vidas, sendo que 7,5 milhões apresentarão ocorrências graves e agudas (SCANDOLARA, et al., 2015).

Cavalheiro e Tolfo (2011) pensam que o trabalho pode ser compreendido como fonte de prazer e realização, visto que ele representa o que há de mais humano no homem: sua subjetividade, entretanto exista também seus contrapontos, pois o trabalho evolui como elemento provocador tanto de prazer quanto de sofrimento.

Para Batista et al. (2013), a satisfação e o desprazer decorrentes de atividades do trabalho possuem relação com a carga psíquica que tal tarefa proporciona. Desse modo, com o decorrer do tempo essa insatisfação diária, na maioria das vezes, pode causar o aparecimento de transtornos mentais em trabalhadores mais suscetíveis.

Reconhece-se que a depressão está associada à diminuição da produtividade e, consequentemente do desempenho no trabalho, limitando a contribuição que o indivíduo acometido pela doença poderia dar à sociedade, ocasionando um impacto negativo na vida do mesmo (PEREIRA; MORGADO, 2012). Estudos apontam a depressão como um fator determinante que envolve a saúde do trabalhador e o seu afastamento das suas atividades diárias (BRAUN; CARLOTTO, 2014).

A exposição ao estresse ininterrupto, relacionado ao trabalho, também compõe um importante fator decisivo dos transtornos depressivos e de outras doenças, tais como: síndrome 
da fadiga crônica e metabólica, síndrome de Burnout, distúrbios do sono e diabetes (SCANDOLARA, et al., 2015).

A depressão e a ansiedade são doenças que podem acometer pacientes que procuram tratamento de suspensão do tabagismo (PAWLINA et al., 2015). Pessoas adultas diagnosticadas com depressão têm mais chance de fumarem; sugere-se este comportamento tem a ver com automedicação, a nicotina, de algum modo, age como calmante ou um mecanismo de relaxamento (ARAÚJO, 2004). Por outro lado, o cigarro é prejudicial à saúde integral do indivíduo e eleva os riscos do aparecimento de diversas doenças.

Outro ponto favorável que será abordado nesta pesquisa se deve a aptidão em executar exercícios físicos, visto que esta influi significativamente na qualidade de vida do indivíduo e pode auxiliar no tratamento e prevenção de quadros depressivos, quando atrelada a outros fatores que completam como fator favorável à melhoria de saúde e Qualidade de Vida dos indivíduos.

O trabalho que ora aqui se desenvolve integra o projeto "Identificando o perfil epidemiológico da Obesidade em Vitória da Conquista” idealizado pelo Núcleo de Extensão, Pesquisa e Estudo de Doenças Crônicas - NEPEdc da Faculdade Independente do Nordeste, e teve como objetivo geral: verificar o índice de funcionários acometidos por depressão em uma instituição de ensino superior em Vitória da Conquista- BA. E como específicos: conhecer o perfil sócio demográfico e epidemiológico desses funcionários; descrever a frequência de hipertensão e tabagismo dos entrevistados; discutir as relações entre o processo de trabalho e caracterizar as ocorrências de depressão que levam servidores docentes e técnicoadministrativos ao afastamento do trabalho; apresentar a importância da atividade física na prevenção e tratamento da depressão. Trata-se de uma pesquisa do tipo analítica com delineamento transversal e abordagem quantitativa e teve como amostra 180 indivíduos do município de Vitória da Conquista- BA.

Este trabalho é importante, pois há necessidade de que os profissionais estejam preparados para realizar uma orientação adequada no tratamento da depressão e que a sociedade conheça os diversos fatores que podem influenciar na evolução da doença e técnicas que possam preveni-las. 


\section{Material e Método}

Este trabalho faz parte do projeto "Identificando o perfil epidemiológico da Obesidade em Vitória da Conquista" idealizado pelo Núcleo de Extensão, Pesquisa e Estudo de Doenças Crônicas - NEPEdc da Faculdade Independente do Nordeste.

Trata-se de uma pesquisa do tipo analítica com delineamento transversal e abordagem quantitativa, que teve como amostra 180 indivíduos do município de Vitória da Conquista-BA. Os dados foram coletados a partir da aplicação de formulário para trabalhadores de instituições deste município, subdivididas em seções: Avaliação cognitiva, Informações sócio demográficas, questionário de atividade física, whoqol-bref, questionário de hábitos alimentares, questionário de doenças pré-existentes, odontológico, avaliação corporal, depressão, ansiedade e tabagismo.

Os dados foram analisados no Programa Estatístico SPSS através de análise descritiva. Para a descrição das variáveis contínuas foram utilizadas a média aritmética, com seu respectivo desvio padrão e para variáveis categóricas, número absoluto e frequência relativa.

O processo de desenvolvimento desse trabalho foi conduzido de acordo com a Resolução 466/12 e submetido à aprovação do Comitê de Ética em Pesquisa da Faculdade Independente do Nordeste - FAINOR.

\section{Resultados e Discussão}

Os funcionários participantes são 54,4\% (F=99) mulheres e 45,6\% homens ( $\mathrm{F}=82)$ com idade média de 37,83 anos. Com relação à classe social, 70\% ( $\mathrm{F}=126)$ dos funcionários se autodeclararam como pertencentes à classe E. Apreendeu-se que quase metade deles apresentam quadro depressivo (43,9\%). Quanto a frequência de hipertensão, os dados mostram que 87,8\% (F=158) não são hipertensos; ao tabagismo, 18,3\% (F=10) não são fumantes e 76,1\% $(\mathrm{F}=137)$ estiveram ausentes no sistema, não responderam a esta questão. Quanto a prática de atividades físicas, 56,1\% ( $\mathrm{F}=101)$ são sedentários. As questões respondidas possibilitaram chegar à conclusão de que a qualidade de vida da maioria $(73,3 \%, \mathrm{~F}=132)$ é normal, enquanto somente $12,8 \%(\mathrm{~F}=23)$ apresentaram nível de qualidade de vida ruim (cf. TABELA 1). 
Tabela 1. Sistematização dos dados sócio demográficos, frequência de depressão, hipertensão, tabagismo, atividade física e qualidade de vida.

\begin{tabular}{|c|c|c|c|}
\hline . & $\mathbf{N}$ & $\mathbf{F}$ & $\%$ \\
\hline \multicolumn{4}{|l|}{ Sexo } \\
\hline & Feminino & 98 & 54,4 \\
\hline & Masculino & 82 & 45,6 \\
\hline
\end{tabular}

Idade

$\begin{array}{ll}\text { Média } & 37,83 \\ \text { Desvio Padrão } & \pm 12,05\end{array}$

Classe social

$\begin{array}{lcc}\text { Classe B } & 3 & 1,7 \\ \text { Classe C } & 19 & 10,6 \\ \text { Classe D } & 28 & 15,6 \\ \text { Classe E } & 126 & 70 \\ \text { Ausentes no sistema } & 4 & 2,1\end{array}$

Frequência de Depressão

$\begin{array}{lcc}\text { Depressivos } & 79 & 43,9 \\ \text { Não depressivos } & 101 & 56,1\end{array}$

\section{Frequência de Hipertensão}

$\begin{array}{lcc}\text { Hipertensos } & 21 & 11,7 \\ \text { Não hipertensos } & 158 & 87,8 \\ \text { Ausentes no sistema } & 1 & 0,5\end{array}$

Tabagismo

Não fumantes $\quad 33 \quad 18,3$

Fumantes $\quad 10 \quad 5,6$

$\begin{array}{lll}\text { Ausentes no sistema } & 137 & 76,1\end{array}$

Atividade física

$\begin{array}{lcc}\text { Não ativo } & 101 & 56,1 \\ \text { Ativo } & 79 & 43,9\end{array}$

Qualidade de Vida

$\begin{array}{lcc}\text { Ruim } & 23 & 12,8 \\ \text { Normal } & 132 & 73,3 \\ \text { Boa } & 25 & 13,9\end{array}$

$\mathbf{F}=$ frequência $\%=$ Percentual em função das respostas dadas

Fonte: Dados colhidos da pesquisa, 2017. 
Embora o percentual encontrado demonstre um equilíbrio entre os gêneros nesta, viuse que dentre os funcionários desta instituição de ensino superior, a maioria dos participantes são do gênero feminino. Em estudo de Braun et al. (2014), aproximadamente 90,0\% dos analisados são mulheres, isto demarca que as mulheres são maioria da população e ocupam, atualmente, mais espaço no mercado de trabalho. Isto confirma que o espaço da mulher na economia nacional tem se ampliado pouco a pouco, pois esta já representa uma boa porcentagem da força de trabalho (MÉNDEZ, 2015).

Este fator se explica com base nas informações da última Pesquisa Nacional por Amostra de Domicílio, divulgada pelo IBGE em 2013; ela mostrou que viviam no Brasil 103,5 milhões de mulheres, o equivalente a $51,4 \%$ da população. Ou seja, no Brasil, as mulheres são maioria da população. Além disso os dados indicam que as mulheres têm tido menos filhos, aumentado a expectativa de vida, ocupado cada vez mais espaço no mercado de trabalho e, hoje em dia, são as responsáveis pelo sustento de 37,3\% das famílias (PORTAL BRASIL, 2015).

A inserção da mulher no mercado de trabalho foi motivada pela necessidade de sua contribuição no ganho financeiro da família; já no início na Revolução Industrial as indústrias absorveram a mão-de-obra feminina a fim de baratear os salários e também pela grande facilidade de disciplinar esse grupo de trabalhadoras (Baylão, 2014). Conforme afirma Correa (2015) a revolução sexual feminina, gerou diminuição na fecundidade e na mudança do papel social das mulheres e aumentou a participação mulheril no mercado de trabalho.

De acordo com o IBGE, a média de vida de um brasileiro é de viver 72,7 anos, isto tem a ver com a quantidade de anos em média que uma determinada população vive (FREITAS,2017). Atualmente, o brasileiro se aposenta, em média, aos 58 anos, de acordo com Marchesan 2017. Os dados colhidos mostraram que idade média apresentada pelos 181 participantes da pesquisa foi de 37,83 anos (DP $\pm 12,05)$, sendo que o participante mais novo apresentou 19 anos de idade e o mais velho 76 anos. Se considerarmos a idade média de aposentadoria dos brasileiros, podemos considerar que estes já passaram da metade da média prevista pela previdência social.

Entende-se por classe social o conceito de que existem distâncias sociais significavas na sociedade. Ou seja, compreende-se que sujeitos e grupos são distintos entre si e ocupam lugares socialmente diferentes (Codato e Leite, 2009). O quadro 1 mostra que a Classificação Econômica no Brasil se distribui em cinco tipos que vai de A a E: Classe A (acima de 20 
salários mínimos), Classe B (de 10 a 20 salários mínimos), Classe C (de 4 a 10 salários mínimos), Classe D (de 2 a 4 salários mínimos) e a Classe E (recebe até 2 salários mínimos).

Quadro 1. Classificação das classes sociais elaborado com base no salário mínimo de 2016.

\begin{tabular}{|c|c|c|}
\hline CLASSE & SALÁRIOS MÍNIMOS (SM) & RENDA FAMILIAR (R\$) \\
\hline A & Acima de $20 \mathrm{SM}$ & $\mathrm{R} \$ 17.600$ ou mais \\
\hline B & 10 a $20 \mathrm{SM}$ & De $R \$ 8.800$ a $R \$ 17.600$ \\
\hline $\mathrm{C}$ & 4 a 10 SM & De $\mathrm{R} \$ 3.520$ a $\mathrm{R} \$ 8.800$ \\
\hline $\mathrm{D}$ & 2 a 4 SM & De $R \$ 1.740$ a $R \$ 3.520$ \\
\hline $\mathrm{E}$ & Até $2 \mathrm{SM}$ & Até $\mathrm{R} \$ 1.760$ \\
\hline
\end{tabular}

Fonte: elaborado pela autora, 2017.

Com relação a classe social, $70 \%(\mathrm{~F}=126)$ dos funcionários se autodeclararam como pertencentes à classe $\mathrm{E}$, ou seja, possuem renda familiar de até $\mathrm{R} \$ 1.760$ (até 2 salários mínimos). É válido ressaltar que o status social é definido de acordo com o nível salarial. Desse modo, pressupõe-se que os salários baixos dos funcionários podem ser o ponto chave na insatisfação com o trabalho, levando ao esgotamento mental e a um possível adoecimento (TERRA, 2010).

Conforme estudo realizado por Dalcin (2009) os baixos salários, enquanto condição precária do trabalho, desencadeiam o surgimento de estado de estresse. Neste mesmo estudo, constatou-se que 8 a cada 10 trabalhadores entrevistados precisam de alguma forma compensar o baixo ganho trabalhando em outros locais para alcançar uma maior renda mensal, enquanto outros economizam em casa, fazendo todas as tarefas domésticas durante a noite e abrindo mão dos momentos de lazer. "A falta de lazer, de habitação e até mesmo a falta de plano de saúde deixa o trabalhador estressado e desesperançoso. Além de não poderem ter uma vida digna, o trabalhador se impossibilita para continuar sua formação profissional" (DALCIN, 2009, p.77).

Segundo estudos na área da psicopatologia do trabalho, a depressão atinge todas as raças, idades e profissões (TEIXEIRA, 2007). As experiências vividas por esses trabalhadores acarretam graves consequências e, muitas vezes, irreparáveis para a saúde e bem-estar físico do trabalhador (BURGARD, 2013). 
Constatou-se nesta pesquisa que quase metade dos profissionais investigados possuem depressão. Ao relacionarmos a ideia média dos entrevistados 37,83 anos (DP $\pm 12,05)$ com a frequência de depressão, podemos afirmar que a ressaltar que a ocorrência de transtornos mentais independe da faixa etária, pois qualquer servidor em qualquer idade pode ser acometido por esta doença. Isto é, a ocorrência de transtornos mentais acontece não somente na terceira idade, mas também na juventude e na idade adulta.

Cavalheiro e Tolfo (2011) assinalam que a depressão pode manifestar-se em diferentes momentos da vida de uma pessoa, sendo compreendida "como qualquer manifestação de tristeza e/ou estresse, ou pode se constituir em um problema de saúde pública, pelo grau de sofrimento e pelas altas taxas de suicídio" (p.243). Desse modo, Menicali (2017) ratifica que o estado depressivo acomete tanto adultos e idosos, quanto crianças e adolescentes; e que isto provocando danos ao desempenho escolar, laboral e/ou ocupacional.

Os dados de prevalência de depressão entre homens e mulheres em dois estudos brasileiros como o de Amato et al. (2010), Cavalheiro e Tolfo (2011) e Lopes et al. (2011) foram significativos no gênero feminino, pois neste houve maior prevalência de desordens depressivas.

Considerando que a maior parte dos participantes dessa pesquisa são do gênero feminino, Cavalheiro e Tolfo (2011) observaram em seus estudos a prevalência de 16\% de depressão nas mulheres (entre grau leve e grave), enquanto os homens não passaram de $8 \%$ (somente grau leve). Também no estudo de Amato et al.(2010) em que se identificou 26,9\% de mulheres depressivas, ultrapassando a prevalência nos homens.

De acordo com a OMS (2011) a probabilidade de uma mulher desenvolver um quadro depressivo ao longo da vida varia de 10 a $25 \%$, enquanto em um homem pode variar de 5 a 12\%. Para Gavin (2013) a probabilidade de as mulheres desenvolverem este tipo de transtorno é maior porque elas são influenciadas por fatores genéticos e psicossociais; este último, ocorre constantemente, visto que o papel da mulher está associado ao trabalho doméstico. A dupla jornada de trabalho compromete, maiormente, a saúde da mulher, além desses aspectos Cavalheiro e Tolfo (2011) destacam questões biológicas, visto que as questões hormonais diferenciam homens e mulheres, as quais as diferenças são relacionadas a faixas etárias (adolescência, período gestacional, tensão pré-menstrual ou menopausa). 
Quanto à influência que os fatores emocionais incidem sobre o quadro de desenvolvimento de hipertensão arterial e a reatividade cardiovascular, infere-se que estes são influenciados por elementos emocionais como impulsividade, resistência, estressores, ansiedade, angústia e raiva (FONSECA, 2009).

No entanto, estudos mais detalhados são necessários para melhor esclarecer essa analogia. Pois no estudo que aqui se delineia o quantitativo de participantes diagnosticados com hipertensão não se assemelham aos quantitativo de depressivos.

Embora ainda ocorra a alta prevalência do tabagismo em alguns países, o número de fumantes tem diminuído mundialmente nas últimas décadas, provavelmente por conta da facilidade ao acesso à prevenção e ao tratamento do tabagismo (PAWLINA et al.,2015; PIPER et al., 2011). Os números desta pesquisa mostraram que a maior parte das respostas a esta pergunta foram ausentes, mas que dos respondentes a maior parte deles $(18,33 \%)$ não possuem o hábito de fumar.

Compreende-se que a ansiedade, depressão, estresse e o baixo nível de motivação são fatores agravantes que podem ser desencadeados nos pacientes em tratamento de combate ao tabagismo (PAWLINA et al., 2015; REICHERT et al., 2008). A substância nicotina age no funcionamento dos sistemas neurotransmissores e desempenha distintas ações neuroendócrinas que pode influenciar no quadro psicopatológico do paciente em tratamento (HERRÁN et al., 2000).

Esta pesquisa mostrou que a maior parte dos participantes não praticam exercícios físicos. De acordo com Oliveira (2014) este tipo de prática destaca-se no tratamento da depressão tanto leve quanto moderada; ela influencia com valor preventivo e como tratamento, através dos mecanismos psicológicos e/ou biológicos. Assim, o hábito de praticar exercícios físicos é um fator benéfico frente aos transtornos emocionais, pois compreende-se que a realização de exercícios físicos ocasiona a liberação de dopamina e endorfinas pelo organismo, gerando um efeito relaxante, que após o esforço mantém o estado de equilíbrio psicoemocional mais constante frente adversidades (BORSON, 2010; BOCALINI et al., 2010).

Mayer (2011) salienta que a prática regular de exercício físico proporciona efeito favoráveis a qualidade de vida do indivíduo inibindo o risco de doenças crônico-degenerativas. 


\section{Considerações Finais}

A depressão é vista como um problema de saúde pública e, considerada um dos distúrbios psiquiátricos com alta prevalência, afetando milhões de pessoas em todo o mundo. A insatisfação decorrente de atividades laborais possui relação com a carga psíquica que tais tarefas proporcionam. Assim sendo, com o tempo essa insatisfação diária gera o aparecimento de transtornos mentais em trabalhadores mais suscetíveis.

Observamos neste estudo, que o quantitativo maior de funcionários é do gênero feminino de meia idade e de baixa renda. Nesse interim, acreditamos que os fatores biológicos expõem a grande maioria desses funcionários à possibilidade de desenvolver um quadro depressivo. Fatores externos como a classe social também colaboram com o aparecimento desta patologia, pois pressupõe-se que estes trabalhadores precisam de alguma forma compensar a baixa renda salarial trabalhando em outros locais, o que pode ser o ponto chave na insatisfação com o trabalho, levando ao esgotamento mental. Sugere-se que estudos mais detalhados sejam realizados a fim de discutir as possíveis relações da depressão com o quadro de hipertensos e fumantes. Constatou-se neste estudo, ainda, que a prática de exercícios físicos, através dos mecanismos psicológicos e/ou biológicos, destaca-se no tratamento e prevenção da depressão.

Além disso, quase metade dos funcionários entrevistados apresentou quadro depressivo e a maior parte deles é sedentária. A disfunção psicológica descrita pode ser explicada pelo fato dos mesmos sentirem-se frustrados no momento em que não conseguem emprego na sua área de formação, evidenciando que possuir ensino superior, não é garantia instantânea do sucesso profissional. Mesmo sendo minoria, parte dos indivíduos que compõem a equipe são graduados, interferindo assim, nas variáveis em questão. Uma outra porção destes não cursou esse nível de ensino, atenuando seus sonhos e tendo como consequência os problemas descritos.

\section{Referências}

AMATO, T.C. et al. Trabalho, gênero e saúde mental: uma pesquisa quantitativa e qualitativa entre bombeiros. Cadernos de Psicologia Social do Trabalho, 2010, vol. 13, n. 1, pp. 103118 
APOSTOLO, J. L. A. et al. Depressão, ansiedade e estresse em usuários de cuidados primários de saúde. Rev. Latino-Am. Enfermagem, v. 19, n. 2, p. 348 - 353, 2011.

ARAÚJO, A.J. et al. Diretrizes para Cessação do Tabagismo. J. bras. pneumol. vol.30 suppl.2 São Paulo Aug. 2004.

ARCOS, J.G.; CONSETIVO, J.V.M.; REIA, T.A. Aplicação Da Musculação Em Pessoas com Ansiedade, Depressão e Síndrome do Pânico. Academia Saikoo - Penápolis SP. Lins: SP, 2014.

BATISTA, J. B.V.; CARLOTTO, M. S.; MOREIRA, A. M. Depressão como Causa de Afastamento do Trabalho: Um Estudo com Professores do Ensino Fundamental. Psico, v. 44, n. 2 , p. $257-262,2013$

BAYLÃO, A.L.S. Mulheres são maioria da população e ocupam mais espaço no mercado de trabalho - Portal Brasil. XI Simpósio de Excelência em Gestão e Tecnologia - SEGET, 2014.

BOCALINI, D.S et al.Repercussions of training and detraining by water-based exercise on functional fitness and quality of life: a short term follow-up in healthy older women, Clinics, São Paulo, vol.65 n.12, 2010.

BORSON S. Cognition, Aging and Disabilities: Conceptual Issues.,Physical Medicine \& Rehabilitation Clinics of North America. Vol.21, n.2, p. 375-382, 2010.

BRAUN, A. C.; CARLOTTO, M. S. Síndrome de Burnout: estudo comparativo entre professores do Ensino Especial e do Ensino Regular. Revista Quadrimestral da Associação Brasileira de Psicologia Escolar e Educacional, v. 18, n. 1, p. 125-133, 2014.

BURGARD, S.A.; LIN, K.Y. Bad Jobs, Bad Health? How Work and Working Conditions Contribute to Health Disparities. Am Behav Sci. Aug. 2013.

CAVALHEIRO, G.; TOLFO, S.R. Trabalho e depressão: um estudo com profissionais afastados do ambiente laboral. Psico-USF, v. 16, n. 2, p. 241-249, mai./ago. 2011.

CODATO, A.; LEITE, F.B. Classe social. In: Heloisa Buarque de Almeida; José Szwako. (Org.) Diferenças, igualdade. São Paulo: Berlendis \& Vertecchia, 2009.

CORREA, C.H. Expectativa de Vida no Mercado de Trabalho Brasileiro. Trabalhos para Discussão Brasília n 389 junho 2015 p. 1-47

FONSECA, F.C.A. et al. A influência de fatores emocionais sobre a hipertensão arterial. J Bras Psiquiatr. 2009;58(2):128-134. 
FREITAS, Eduardo de. "Expectativa de vida dos brasileiros "; Brasil Escola. Disponível em $<$ http://brasilescola.uol.com.br/brasil/expectativa-vida-dos-brasileiros.htm>. Acesso em 26 de abril de 2017.

GAVIN, R.J. Depressão, estresse e ansiedade: um enfoque sobre a saúde mental do trabalhador. Dissertação (Mestrado) Departamento de Enfermagem Psiquiátrica e Ciências Humanas da Escola de Enfermagem de Ribeirão Preto da Universidade de São Paulo, 2013.

HERRÁN , A.; et al. - Determinants of smoking behaviour in outpatients with schizophrenia. Schizoph Res 41: 373-81, 2000.

KAWAMURA, et al. Survival rate and causes of mortality in the elderly with depression: a 15year prospective study of a Japanese community sample, the Matsunoyama-Niigata suicide prevention project. Journal of Investigative Medicine, Niigata, v. 55, n.3, p.106-14, 2007.

LIMA, A.F.B.S. ; FLECK, M.P.A. Qualidade de vida e depressão: uma revisão da literatura. Revista psiquiátrica, Rio Grande do Sul, v.31, n.3, 2009.

NASCIMENTO D. C. D.; BRITO M. A C.; SANTOS A. D. Depressão em idosos residentes em uma instituição asilar na cidade de Juazeiro do Norte, Ceará, Brasil. J. Manag. Prim. Health Care, v. 4, n. 3, p. 146 - 150, 2013.

PAWLINA, M.M.C. et al. Depressão, ansiedade, estresse e motivação em fumantes durante o tratamento para a cessação do tabagismo. J Bras Pneumol. 2015;41(5):433-439.

PEREIRA, M.M.;MORGADO, M.A. A saúde do trabalhador em registros do INSS de Mato Grosso: processos de adoecimento psíquico por motivo de trabalho. Revista Anagrama, 5(4), 22-29, 2012.

PITCHOT, W. et al., Recovering from depression: a matter of objective and determination. Revue Medicale de Liege, v. 65, n. 5 - 6, p. 370 - 380, 2010.

SCANDOLARA, T. B.; WIETZIKOSKI, E. C.; GERBASI, A. R. V.; SATO, S. W. Avaliação dos níveis de estresse e depressão em professores da rede pública do município de Francisco Beltrão - PR. Arq. Cienc. Saúde UNIPAR, Umuarama, v. 19, n. 1, p, 31-38, jan./ abr. 2015.

PORTAL BRASIL. Mulheres são maioria da população e ocupam mais espaço no mercado de trabalho - Portal Brasil. (2015). Disponível em: http://www.brasil.gov.br/cidadania-ejustica/2015/03/mulheres-sao-maioria-da-populacao-e-ocupam-mais-espaco-no-mercado-detrabalho Acesso em Abril de 2017.

MARCHESAN, R. De cada três aposentados, dois ganham um salário mínimo. Do UOL, em São Paulo 18/01/2017 Disponível em: https://economia.uol.com.br/noticias/redacao/2017/01/18/brasileiro-se-aposenta-aos-58-emmedia-e-a-maioria-ganha-1-salario-minimo.htm. Acesso em 26 de Abril de 2017. 
MAYER F et al. The Intensity and Effects of Strength Training in the Elderly. Deutsches Ärzteblatt International,vol. 108, n.21, p. 359-64, 2011.

MÉNDEZ, D.M. The impact of Economic liberalization on Gender equality in Colombia. CS no.15 Cali Jan./April 2015. Disponível em: http://www.scielo.org.co/scielo.php?script=sci_arttext\&pid=S2011-03242015000100007 Acesso em Maio de 2017.

MENICALI, D. Depressão: adultos, jovens e crianças podem ter sintomas. Jundiaí Agora, 2017. Disponível em: http://www.jundiagora.com.br/depressao-sintomas/ Acesso em Abril de 2017.

OLIVEIRA, V.I.M. Depressão e atividade física. Confef, Março de 2014. http://www.listasconfef.org.br/comunicacao/banco_de_ideias/VivianeOliveira.pdf

OMS. Estadísticas sanitarias mundiales 2011. Organização Mundial de Saúde, 2011. Disponível em: .http://www.who.int/whosis/whostat/ES_WHS2011_Full.pdf Acesso em Abril de 2017.

PIPER M.E. et al. Anxiety diagnoses in smokers seeking cessation treatment: relations with tobacco dependence, withdrawal, outcome and response to treatment. Addiction. $2011 ; 106(2): 418-27$.

REICHERT J. et al. Smoking cessation guidelines--2008. J Bras Pneumol. 2008;34(10):84580 .

\section{Como citar este artigo (Formato ABNT):}

OLIVEIRA, Daiana C.; SILVA, Matheus L.; SOARES, Fernanda de Jesus; SAMPAIO, sara C.; BITTENCOURT, Felipe O.; DUARTE, Stênio F. P. Avaliação da Depressão em Funcionários de uma Instituição de Ensino Superior Particular em Vitória Da Conquista-BA. Id on Line Revista Multidisciplinar e de Psicologia, Maio de 2017, vol.11, n.35, p. 392-404. ISSN: 1981-1179.

Recebido: 25.05 .2017

Aceito: 27.05 .2017 